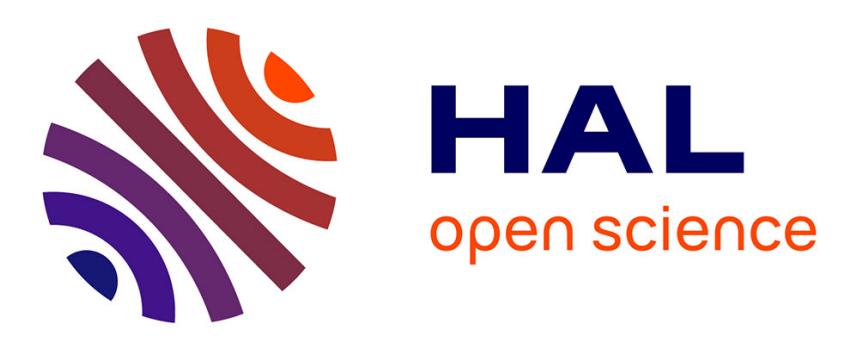

\title{
Un modèle supralexical de représentation de la morphologie dérivationnelle en français
}

Hélène Giraudo

\section{To cite this version:}

Hélène Giraudo. Un modèle supralexical de représentation de la morphologie dérivationnelle en français. Annee Psychologique, 2005, 1 (105), pp.171-195. 10.3406/psy.2005.3825 . hal-02434822

\section{HAL Id: hal-02434822 \\ https://hal.science/hal-02434822}

Submitted on 10 Jan 2020

HAL is a multi-disciplinary open access archive for the deposit and dissemination of scientific research documents, whether they are published or not. The documents may come from teaching and research institutions in France or abroad, or from public or private research centers.
L'archive ouverte pluridisciplinaire HAL, est destinée au dépôt et à la diffusion de documents scientifiques de niveau recherche, publiés ou non, émanant des établissements d'enseignement et de recherche français ou étrangers, des laboratoires publics ou privés. 


\section{H. Giraudo}

\section{Un modèle supralexical de représentation de la morphologie dérivationnelle en français}

In: L'année psychologique. 2005 vol. 105, n¹. pp. 171-195.

Citer ce document / Cite this document :

Giraudo H. Un modèle supralexical de représentation de la morphologie dérivationnelle en français. In: L'année psychologique. 2005 vol. 105, n¹. pp. 171-195.

doi : 10.3406/psy.2005.3825

http://www.persee.fr/web/revues/home/prescript/article/psy_0003-5033_2005_num_105_1_3825 


\section{Résumé}

\section{Résumé}

La plupart des travaux actuels tendent à donner aux informations morphologiques un rôle essentiel du point de vue de l'accès lexical, néanmoins de nombreuses controverses subsistent concernant la façon où ces informations sont représentées en mémoire à long terme. Si l'on se place dans le cadre de modèles hiérarchiques de la reconnaissance de mots, dans lesquels les codes lexicaux sont traduits en codes orthographiques et ou phonologiques décrivant les mots entiers, puis en codes sémantiques, deux localisations peuvent être proposées pour des représentations morphologiques : avant les représentations de mots (hypothèse sublexicale) ou après ces représentations (hypothèse supralexicale). Selon l'hypothèse sublexicale, la reconnaissance d'un mot engendre systématiquement une procédure d'analyse de ses constituants morphémiques avant que celui-ci ne puisse être identifié selon sa forme globale. En revanche, selon l'hypothèse supralexicale, ce sont les représentations globales des mots qui, une fois activées, permettent l'activation des représentations morphémiques situées à un niveau supérieur, ces dernières imposant ainsi aux représentations lexicales une organisation en termes de familles morphologiques. Cet article présente un ensemble de données récentes, obtenues en français et à l'aide du paradigme d'amorçage masqué associé à la tâche de décision lexicale, qui suggèrent que les informations morphologiques dérivationnelles sont représentées à un niveau supralexical de traitement.

Mots clés : reconnaissance de mots, information morphologique, modèle supralexical

\section{Abstract}

Summary : A supralexical model of morphological representation for French derivational morphology

There is a general consensus among psycholinguists today that morphological information is explicitly represented in the mental lexicon and exploited during language processing. However, there is still much controversy surrounding the way such information is represented. In a generic hierarchically organized model of word recognition where sublexical codes are mapped onto whole-word orthographic and/or phonological codes, and from there to semantics, there are two possible locations for morphemic representations : below whole-word representations (the sublexical hypothesis), or above the wholeword level (the supralexical hypothesis). According to the sublexical hypothesis, a word stimulus is first parsed into its morphological components before the word can be recognized as a whole. According to the supralexical hypothesis, morphemic representations are contacted after whole-word representations and in this way impose an organization on lower-level form representations in terms of morphological families. Recent data obtained in French and using the priming paradigm associated with the lexical decision task are presented in support of a supralexical model of morphological representation for French derivational morphology.

Key words : word recognition, morphological information, supralexical model. 


\title{
NOTE THÉORIQUE
}

\author{
Université de Provence et CNRS \\ Laboratoire Parole et Langage ${ }^{1}$
}

\section{UN MODÈLE SUPRALEXICAL DE REPRÉSENTATION DE LA MORPHOLOGIE DÉRIVATIONNELLE EN FRANÇAIS} Hélène Giraudo*

SUMMARY : A supralexical model of morphological representation for
French derivational morphology

There is a general consensus among psycholinguists today that morphological information is explicitly represented in the mental lexicon and exploited during language processing. However, there is still much controversy surrounding the way such information is represented. In a generic hierarchically organized model of word recognition where sublexical codes are mapped onto whole-word orthographic and/or phonological codes, and from there to semantics, there are two possible locations for morphemic representations : below whole-word representations (the sublexical hypothesis), or above the whole-word level (the supralexical hypothesis). According to the sublexical hypothesis, a word stimulus is first parsed into its morphological components before the word can be recognized as a whole. According to the supralexical hypothesis, morphemic representations are contacted after wholeword representntions and in this way impose an organization on lower-level form representations in terms of morphological families. Recent data obtained in French and using the priming paradigm associated with the lexical decision task are presented in support of a supralexical model of morphological representation for French derivational morphology.

Key words : word recognition, morphological information, supralexical model.

* Une partie de ce travail a été soutenue financièrement par une bourse d'études de la Fondation Fyssen.

1. Laboratoire Parole et Langage (CNRs), France, 29, avenue RobertSchuman, 13621 Aix-en-Provence cedex 1. E-mail : giraudo@up.univ-aix.fr 


\section{INTRODUCTION}

La majeure partie des recherches menées dans le cadre de la reconnaissance de mots a pour objectif de spécifier, outre la nature du traitement effectué au niveau des structures lexicales dans la compréhension de mots, l'architecture fonctionnelle du lexique mental. Les travaux élaborés dans ce cadre de recherche s'intéressent au codage et à la représentation en mémoire à long terme des informations orthographiques, phonologiques, morphologiques et sémantiques impliquées lors de la perception du langage. À l'heure actuelle, l'interprétation des données empiriques s'effectue principalement dans le cadre de modèles d'activation interactive connexionnistes décrivant la structure des modules de traitement de la lecture, pour lesquels les différents types d'unités de traitement (lettres, phonèmes, syllabes, formes de mots, concepts) sont représentés de façon localisée (par opposition avec une vue distribuée du traitement lexical, voir Grainger et Jacobs, 1998 pour une discussion). Cette note théorique se propose de montrer que, parmi les différentes représentations possibles des unités morphologiques dans le lexique, un modèle qui inclut un niveau de traitement morphologique à l'interface des niveaux lexical et sémantique permet le mieux de rendre compte des données empiriques obtenues récemment en français. La première partie de cette note théorique expose les différentes alternatives permettant de rendre compte du traitement morphologique des mots complexes. La seconde partie présente un ensemble de données récentes qui ont été obtenues en français et qui suggèrent que l'information morphologique est représentée en mémoire à l'interface des niveaux de traitement lexical et sémantique.

L'étude du codage des informations morphologiques (décrites par les linguistes comme étant impliquées dans les mécanismes syntaxiques permettant la constitution des phrases et dont la combinaison correspond au processus de formation des mots) a donné lieu à un vaste ensemble de travaux expérimentaux qui ont eu pour objectif de déterminer si l'information morphologique contenue dans les mots morphologiquement complexes (i.e., constitués d'au moins deux morphèmes tels que le mot chanteur $=[$ chant -$]+[$-eur $]$ ) était utilisée par le lecteur lors de leur identification. Au travers de diverses langues, à l'aide de divers paradigmes expérimentaux et divers indices de traitement, il a été clairement mis en évidence que l'information morphologique était utilisée par le lecteur lors de la reconnaissance de mots isolés (e.g., Beauvillain, 1996 ; Boudelaa et Marslen-Wilson, 2001 ; Colé, Segui et Taft, 1997 ; Drews et Zwitserlood, 1995 ; Deutsch, Frost, Pollatsek et Rayner, 2000 ; Feldman, 1991 ; Grainger, Colé et Segui, 1991 ; Holmes et O'Reagan, 1992 ; Meunier et Segui, 1999 ; Marslen-Wilson, Komisarjevski-Tyler, Waksler et Older, 1994; Taft et Forster, 1975).

S'il est à l'heure actuelle largement admis que l'information morphologique est utilisée lors de la reconnaissance de mots, une caractéristique de 
ce domaine de recherche réside dans la diversité des hypothèses formulées à l'égard du mode d'accès et de représentation de cette information en mémoire à long terme. Trois hypothèses principales peuvent néanmoins être dégagées de cet ensemble de propositions. La première hypothèse suggère que tous les mots morphologiquement simples ou complexes sont accédés et représentés globalement. L'information morphologique est alors utilisée uniquement lors de la reconnaissance de mots complexes nouveaux (Butterworth, 1983 ; Manelis et Tharp, 1977). La seconde hypothèse, appelée hypothèse décompositionnelle, propose, à l'inverse, que les mots morphologiquement complexes sont identifiés via un processus systématique de décomposition morphologique prélexical qui consiste à isoler la racine de l'affixe, l'accès lexical s'effectuant sur la base de l'identification de cette racine (Taft et Forster, 1975). Enfin la troisième hypothèse suggère que les représentations lexicales des mots morphologiquement complexes peuvent être contactées selon deux voies d'accès : l'une basée sur la forme globale des mots et l'autre sur ses constituants morphologiques. L'activation en parallèle de ces deux voies permet de contacter le lexique mental constitué de représentations orthographiques, morphologiquement décomposées (e.g., Caramazza, Laudanna et Romani, 1988 ; Frauenfelder et Schreuder, 1992 ; Schreuder et Baayen, 1995 ; voir aussi Deutsch, Frost et Forster, 1998).

\section{LES EFFETS D'AMORCAGE MORPHOLOGIQUE}

LORS DE LA RECONNAISSANCE VISUELLE DE MOTS

De nombreux travaux expérimentaux ont utilisé le paradigme d'amorçage associé à la tâche de décision lexicale ou de dénomination afin d'étudier la façon dont l'information morphologique était représentée en mémoire. Le paradigme d'amorçage consiste en la présentation d'un stimulus amorce (masqué ou non et durant une durée variable) qui partage un certain nombre de traits linguistiques avec un mot appelé cible, présenté subséquemment et sur lequel les sujets doivent effectuer une tâche d'identification donnée. Ce paradigme, qui permet de manipuler de façon précise les relations linguistiques qui sont partagées entre l'amorce et la cible, offre ainsi la possibilité d'examiner l'effet excitateur ou inhibiteur de l'activation d'une information d'un premier mot sur le traitement subséquent d'un second. Dans ce cadre, l'effet d'amorçage peut être compris comme un phénomène de transfert d'activation des propriétés linguistiques du mot amorce vers le mot cible. Ce transfert d'activation est supposé opérer via l'activation de représentations communes partagées qui créent une connexion de nature excitatrice ou inhibitrice entre la représentation du mot amorce et celle du mot cible.

Parce que, dans la plupart des langues romanes, le partage d'un morphème entre deux mots (e.g., laitier et laifage) implique que ceux-ci parta- 
gent à la fois des traits orthographiques/phonologiques et des traits sémantiques, l'utilisation du paradigme d'amorçage est un des outils privilégiés des psycholinguistes car il leur permet de différencier les effets relevant du partage d'une information morphologique (e.g., laitier-laitage) d'effets qui résultent du partage d'informations d'ordre formel (e.g., laitue-laitage) ou sémantique (e.g., yaourt-laitage). D'une manière générale, les travaux qui ont utilisé l'amorçage, qu'il s'agisse du paradigme d'amorçage immédiat classique (où la durée de présentation de présentation de l'amorce est supérieure à $200 \mathrm{msec}$ ) ou du paradigme d'amorçage masqué (développé par Forster et Davis en 1984 et où la durée de présentation de présentation de l'amorce est inférieure à $60 \mathrm{msec}$ ), ont mis en évidence que les effets d'amorçage morphologique étaient de nature facilitatrice et se distinguaient d'effets de nature purement orthographique/phonologique (Deutsch et al., 1998 ; Drews et Zwitserlood, 1995 ; Feldman et Bentin, 1994 ; Giraudo et Grainger, 2000 ; Grainger et al., 1991 ; Pastizzo et Feldman, 2002 ; Marslen-Wilson et al., 1994; Napps et Fowler, 1987) et d'effets de nature purement sémantique (Bentin et Feldman, 1990 ; Feldman et Soltano, 1999 ; Frost, Deutsch, Gilboa, Tannenbaum et Marslen-Wilson, 2000 ; Marslen-Wilson et al., 1994 ; Napps, 1989 ; Rastle, Davis, MarslenWilson et Tyler, 2000 ; Stolz et Besner, 1998). En outre, certains résultats suggèrent qu'aucun effet d'amorçage sémantique ne peut être observé lorsque l'on utilise le paradigme d'amorçage masqué associé à une tâche de décision lexicale (Frost et al., 1997 ; Rastle et al., 2000).

Récemment, un ensemble de travaux expérimentaux ayant utilisé le paradigme d'amorçage masqué a proposé que le traitement des mots morphologiquement complexes impliquait des unités morphémiques bien distinctes des représentations formelles (orthographiques/phonologiques) et sémantiques des mots (Colé et al., 1997 ; Deutsch, Frost et Forster, 1998 ; Drews et Zwitserlood, 1995 ; Frost, Deutsch et Forster, 1999 ; Frost, Deutsch et Forster, 1997 ; Giraudo et Grainger, 2001 ; Grainger et al., 1991 ; Longtin, Segui et Halle, 2003 ; Pastizzo et Feldman, 2002 ; Schriefers, Zwitserlood et Roelofs, 1991 ; Stolz et Besner, 1998 ; Taft, 1994 ; Zwitserlood, 1994). Cependant, si l'on admet que les unités morphologiques sont représentées de façon explicite et indépendante au sein de l'architecture du lexique mental, il reste à déterminer le locus précis de ces unités par rapport aux autres unités de représentation. À cet effet, deux possibilités de représentation peuvent être envisagées.

\section{DEUX ARCHITECTURES CONNEXIONNISTES DU TRAITEMENT DE L'INFORMATION MORPHOLOGIQUE}

L'interprétation de données empiriques issues de l'étude des phénomènes morphologiques peut être effectuée dans le cadre de deux architectures (correspondant à deux extensions possibles du modèle connexionniste 
d'activation interactive proposé par McClelland et Rumelhart en 1981) qui dérivent directement de deux des trois hypothèses représentationnelles proposées dans la littérature. Ces deux architectures sont constituées de plusieurs niveaux de représentation séparés, contenant des représentations explicites qui décrivent les mots d'un point de vue formel, sémantique et morphologique. À l'intérieur de chaque niveau, les représentations sont reliées entre elles par des liens inhibiteurs, les connexions inter-niveaux sont bidirectionnelles et excitatrices. Les deux modèles diffêrent du point de vue du locus de représentation des unités morphémiques : ce niveau morphémique se situe soit à l'interface du niveau de représentation lexicale (description orthographique/phonologique des mots) et du niveau de représentation sémantique (modèle supralexical), soit avant le niveau lexical (modèle sublexical).

La première hypothèse d'un « accès direct » propose que l'accès aux représentations des mots morphologiquement complexes est médiatisé par l'activation de la forme globale des mots, sans tenir compte de leurs constituants morphémiques. Dans ce cadre, chaque mot possède une représentation lexicale particulière et indépendante (Butterworth, 1983 ; Manelis et Tharp, 1977). Les régularités inhérentes à la formation des mots sont codées dans le lexique comme des informations optionnelles ou supplémentaires et utilisées dans le cas d'un échec à l'accès global du mot ou encore dans le cas de mots complexes non familiers ou nouveaux. Une extension logique de cette hypothèse à un modèle connexionniste d'activation interactive consiste en l'ajout d'un niveau de représentation supralexical de la morphologie, se situant à l'interface des représentations lexicales et des représentations sémantiques des mots (cf. fig. 1).

Dans le modèle supralexical (développé par Giraudo et Grainger, 2000), la présentation d'une série de lettres engendre l'activation d'unités correspondant à des descriptions orthographiques/phonologiques de mots. Les unités qui contiennent un ou plusieurs morphèmes sont connectées à un niveau morphologique qui contient des affixes et des racines. Dès qu'un mot complexe ou qu'une racine libre est présenté(e) à l'entrée du système, l'activation des représentations lexicales pouvant correspondre à l'entrée va automatiquement engendrer l'activation des morphèmes que ces représentations contiennent, lesquelles en retour vont activer l'ensemble des unités lexicales qui leur sont reliées. Par conséquent, dans ce type de modèle, les unités morphémiques permettent d'organiser les représentations lexicales formelles en familles morphologiques.

La seconde hypothèse, appelée hypothèse de décomposition morphologique prélexicale (développée dans le modèle verbal de Taft et Forster en 1975), décrit la mise en cuvre d'une analyse en « aveugle » systématique des constituants morphémiques possibles d'un mot avant l'accès au lexique proprement dit. Cette procédure de décomposition morphologique des mots complexes consiste à isoler l'affixe de la racine (que les auteurs nomment littéralement de procédure d' "affix stripping ») afin de contac- 


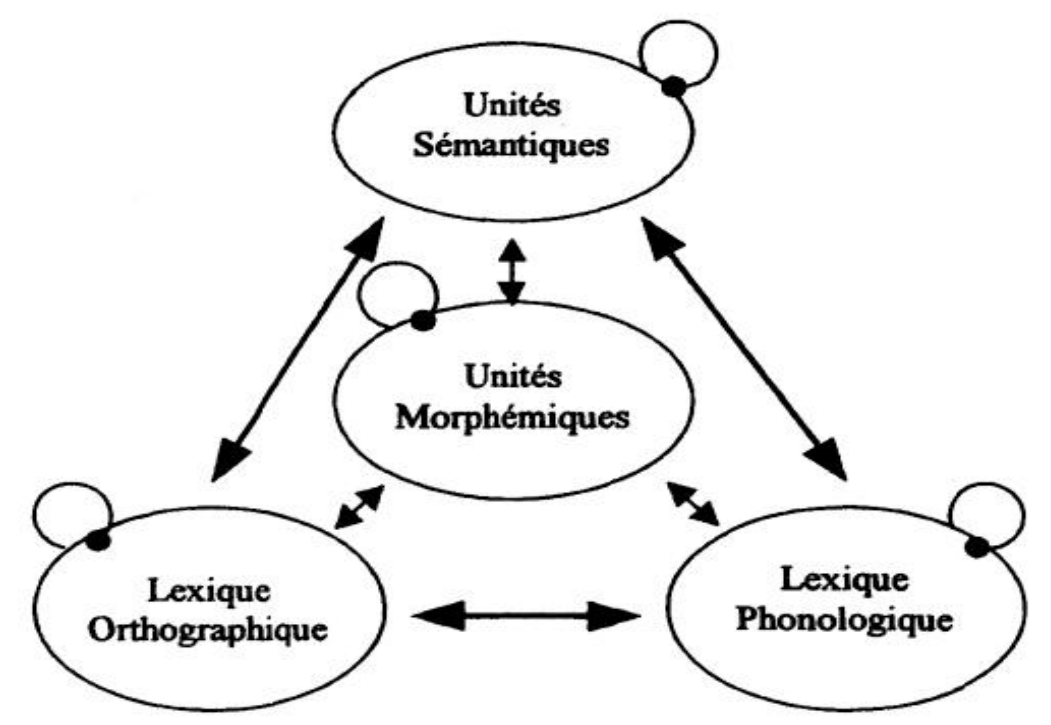

Fig. 1. - Proposition d'architecture pour un modèle d'activation interactive connexionniste implémentant un niveau supralexical de représentation morphologique. Les unités de même niveau sont reliées entre elles par des connexions inhibitrices $(-\bullet)$, des connexions bidirectionnelles facilitatrices relient les unités de niveaux différents $(\leftrightarrow)$

Proposed architecture for a connectionist model implementing a supralexical level of morphological representation. Units at the same level are linked by inhibitory connection, bi-directional facilitory connections link units at different levels

ter la représentation lexicale de cette dernière qui constitue l'entrée lexicale de tous les mots affixés partageant cette racine. Une des implications majeures de ce modèle est que tout mot qui comprend une séquence de lettres pouvant correspondre à un morphème induit une procédure d'analyse morphologique même si cette séquence de lettres correspond en réalité à un pseudo-morphème ${ }^{1}$.

La rigidité d'une telle procédure de décomposition en « aveugle » automatique remet fortement en cause la valeur écologique de ce type de modèle et Taft a plus récemment (1994) proposé une adaptation de son modèle de décomposition prélexicale en un modèle d'activation interactive. Le modèle (cf. fig. 2) incorpore un niveau de représentation morphémique prélexical, de sorte que l'activation d'une représentation lexicale est médiatisée par l'activation préalable de ses constituants morphémiques. La nouveauté de ce modèle réside dans le fait que seuls les mots morphologiquement complexes sont accédés selon l'activation de leurs morphèmes

1. Un pseudo-morphème est une partie de mot qui est identique à un morphème mais qui n'en a pas la fonction, tel que [lait-] dans [laitue] n'est pas un morphème racine alors que [lait-] dans [laitier] en est un. 


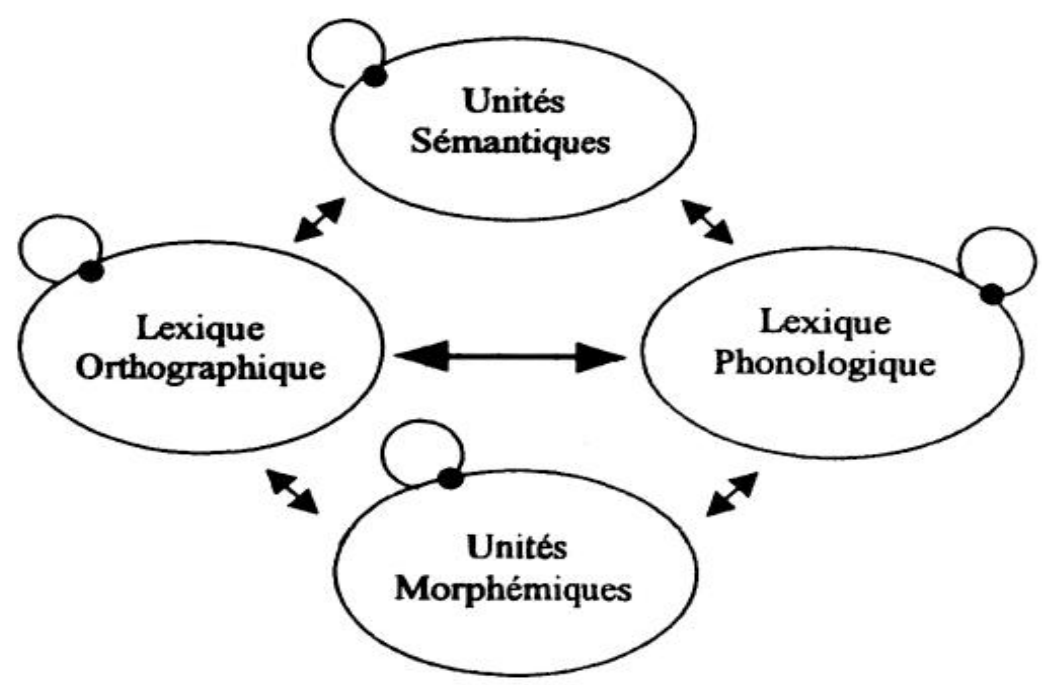

Fig. 2. - Proposition d'architecture pour un modèle d'activation interactive connexionniste implémentant un niveau sublexical de représentation morphologique. Les unités de même niveau sont reliées entre elles par des connexions inhibitrices $(-\bullet)$, des connexions bidirectionnelles facilitatrices relient les unités de niveaux différents $(\leftrightarrow)$

Proposed architecture for a connectionist model implementing a sub-lexical level of morphological representation. Units at the same level are linked by inhibitory connection, bi-directional facilitory connections link units at different levels

constituants, du moins durant leur reconnaissance consciente (nous reviendrons plus tard sur ce point dans le texte).

Dans ce type d'architecture, la présentation d'un mot morphologiquement complexe à l'entrée du système engendre dans un premier temps l'activation de ses morphèmes constituants (i.e., affixe et racine) au niveau des unités morphémiques de traitement. Ces unités morphémiques vont ensuite activer l'unité mot qui contient ces mêmes morphèmes mais aussi l'ensemble des unités mots qui lui sont morphologiquement reliées. Dans ce cadre, les unités morphémiques correspondent à des unités d'accès au lexique.

Dans les deux modèles de représentation de la morphologie, les unités morphologiques sont explicitement représentées de façon séparée des autres unités de traitement, ce qui implique qu'elles constituent le siège de tout effet morphologique observé. Par conséquent, ces deux modèles prédisent des effets morphologiques qui vont dépendre directement du locus des unités morphémiques par rapport aux autres unités de traitement. L'ensemble des travaux expérimentaux présentés dans cette note théorique a testé les prédictions respectives de ces deux modèles afin de déterminer quelle était l'architecture qui permettait le mieux de rendre compte des effets morphologiques. 
Les travaux présentés ont été réalisés en français et ont utilisé le paradigme d'amorçage masqué (durée d'exposition de l'amorce égale à $57 \mathrm{msec}^{1}$ ) associé à la tâche de décision lexicale.

\section{INFLUENCE DES RACINES ET PSEUDO-RACINES SUR LE TRAITEMENT DE MOTS SUFFIXES DERIVES}

Le paradigme d'amorçage masqué permet d'étudier de façon précise la nature des relations partagées entre deux mots reliés. En d'autres termes, ce paradigme permet d'examiner le partage de représentations spécifiques entre les représentations de mots ainsi que la nature excitatrice ou inhibitrice des connexions qui les relient à l'intérieur du lexique mental. Les modèles du traitement morphologique que nous avons décrits plus haut supposent tous deux que les effets d'amorçage morphologique facilitateurs résultent de la préactivation de l'unité morphémique que le mot amorce partage avec le mot cible. La préactivation de cet élément morphémique permettrait une identification plus rapide de la cible par rapport à une condition d'amorçage non reliée.

Dans le modèle supralexical de représentation de la morphologie, les unités morphémiques sont situées au-dessus des unités mots. La présentation d'un mot morphologiquement relié en amorce va activer l'ensemble des unités mots contenant des lettres communes avec le stimulus présenté en entrée (i.e., les mots qui lui sont morphologiquement reliés mais aussi ses voisins orthographiques). Toutefois seules les unités mots reliées morphologiquement au stimulus visuel vont pouvoir alors activer leurs morphèmes constituants situés au niveau morphologique supérieur. Ces unités morphémiques vont en retour exercer un effet d'excitation sur les unités mots contenant ces dernières. L'identification subséquente du mot cible sera donc facilitée car l'inhibition induite par le mot amorce sur l'ensemble de ses voisins orthographiques à un même niveau (qu'ils soient morphologiquement reliés ou pas) aura été réduite par l'excitation descendante provenant de l'unité morphémique que le mot amorce partage avec le mot cible. Dans ce cadre, l'effet de facilitation morphologique résulte, d'une part, d'un effet d'inhibition latérale entre les représentations de mots et, d'autre part, d'un effet d'excitation descendant compensatoire provenant d'une unité morphémique qui s'exerce sur les représentations orthographiques/phonologiques des mots qui lui sont morphologiquement reliées. Parce que l'accès au niveau lexical n'est ici pas médiatisé par l'activation préalable de représentations morphologiques, le modèle ne prédit pas d'effets différents en fonction de la nature simple ou complexe du mot amorce.

1. Une durée d'exposition de l'amorce inférieure à 60 msec peut être considérée comme infraliminaire pour tout participant (cf. Forster et Davis, 1984). 
Dans le modèle sublexical, les unités morphémiques sont contactées avant les unités mots. Les effets morphologiques facilitateurs s'expliquent donc en termes d'emprunt répété d'une même représentation morphologique qui tient lieu d'unité d'accès. La présentation d'un mot amorce morphologiquement relié à la cible va activer dans un premier temps les unités morphémiques qui le constituent et ces unités vont en retour activer les représentations de mots constituées de ces mêmes unités morphémiques. L'identification subséquente du mot cible sera donc facilitée par la préactivation de l'un de ses morphèmes constituants.

Par ailleurs, ce modèle suppose que, si les morphèmes constituent les unités d'accès aux représentations globales des mots morphologiquement complexes, la présentation d'une racine libre reliée devrait produire un effet de facilitation sur la reconnaissance d'un mot cible morphologiquement complexe plus important que la présentation d'un mot complexe relié. En effet, le coût computationnel du traitement d'une racine libre amorce devrait être beaucoup moins important que celui occasionné par un mot morphologiquement complexe qui, présenté à l'entrée du système, va activer deux unités morphémiques, au lieu d'une seule dans le cas d'une racine libre reliée.

Les prédictions respectives des approches supralexicale et sublexicale de représentation de l'information morphologique ont été testées au travers d'une série d'expérimentations utilisant le paradigme d'amorçage masqué associé à la tâche de décision lexicale (Giraudo et Grainger, 2001). Les résultats de ces expériences ont permis de montrer que, par rapport à une condition d'amorçage non reliée, les amorces racines produisent un effet de facilitation sur le traitement de mots suffixés cibles d'amplitude équivalente à celui induit par des mots suffixés amorces. Par ailleurs, on a pu observer que seules les amorces réellement morphologiquement complexes pouvaient produire des effets de facilitation sur le traitement des cibles. En effet, les amorces monomorphémiques contenant une pseudo-racine ne semblent pas avoir eu d'influence sur les performances de traitement des mots cibles et ne différaient pas des amorces non reliées (cf. fig. 3 et 4).

Ces données ne semblent pas vérifier les prédictions que l'on peut dégager du modèle sublexical du traitement morphologique puisque, de par le locus des unités morphémiques, ce modèle prédit, au contraire, des effets de facilitation plus importants (en termes d'amplitude) lorsque le mot amorce relié correspond à la racine libre d'une cible morphologiquement complexe que lorsque le mot amorce est un mot complexe. En effet, la présentation d'un mot amorce complexe devrait occasionner un coût computationnel de traitement plus important que celui occasionné par le traitement d'une racine libre, pour laquelle aucune procédure de décomposition morphologique prélexicale n'est nécessaire. Or les résultats ont montré que l'amplitude de l'effet de facilitation produit par une amorce correspondant à une racine libre ne différait pas de celui produit par une amorce correspondant à un mot suffixé (cf. fig. 3). Par ailleurs, toujours selon le modèle 

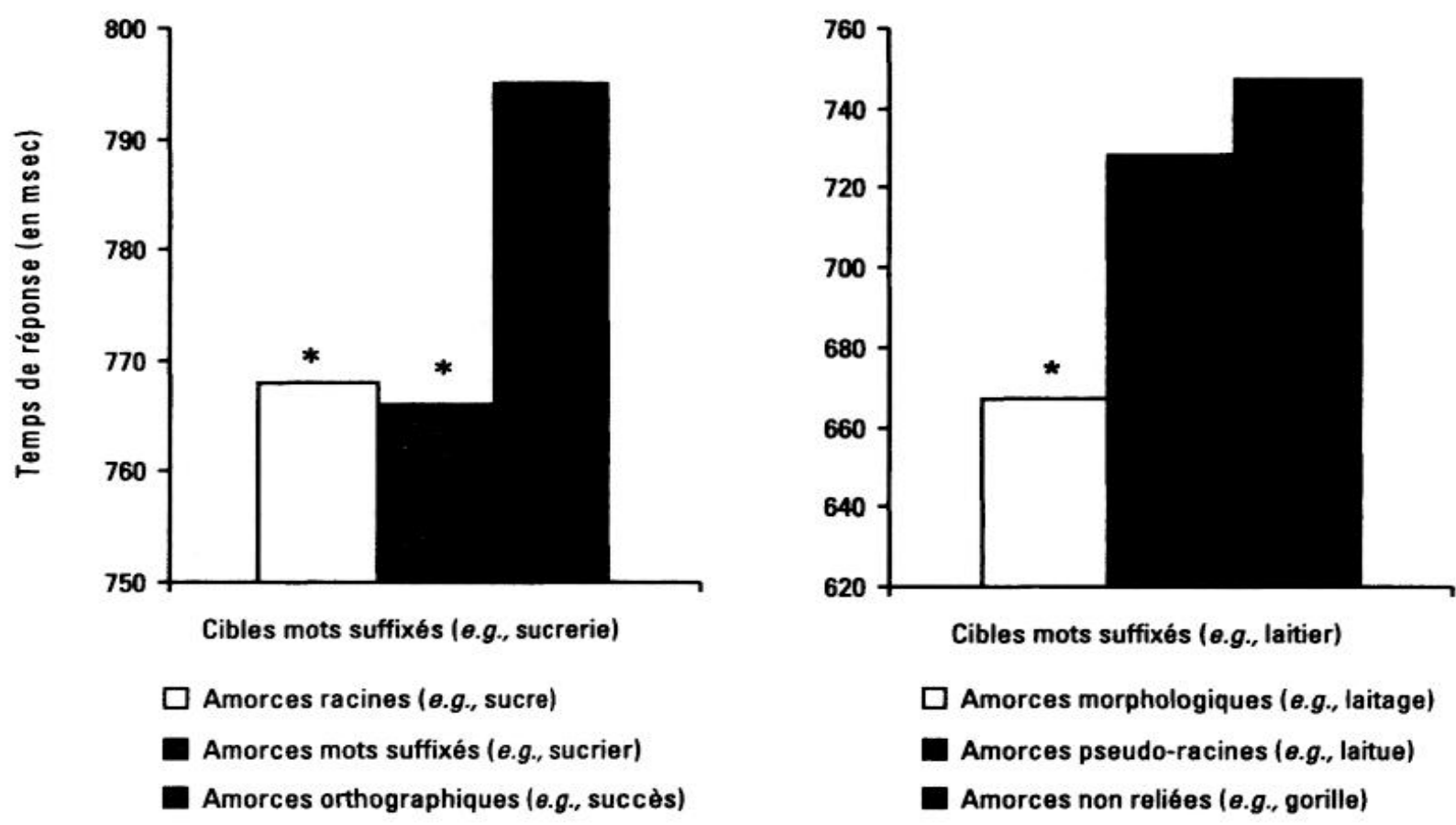

Fig. 3-4. - Principaux résultats obtenus par Giraudo et Grainger (2001).

Latences de décision lexicale sur des mots cibles dérivés testés dans le paradigme d'amorçage masqué

utilisant un temps d'exposition de l'amorce de 57 msec

* indique un effet d'amorçage significatif par rapport à la condition contrôle - orthographique ou non reliée - $(p<.05$ par sujet et par item)

Main results obtained by Giraudo et Grainger (2001).

Lexical decision times for derived target words tested in masked priming paradigm using 57 msec primes

* indicates a significant priming effect compared to the spelling or un-related control condition ( $p<.05$ by subject and by item)

sublexical, des conditions d'amorçage bref et masqué auraient dû favoriser, l'émergence d'effets d'amorçage positifs dans la condition où les mots amorces contiennent une pseudo-racine puisque l'organisation hiérarchique de ce modèle prédit un traitement prélexical automatique de toute séquence de lettres pouvant correspondre à un morphème. Néanmoins, les résultats que nous avons obtenus ont montré que seuls les mots amorces réellement morphologiquement complexes pouvaient générer des effets d'amorçage, la condition orthographique constituée de mots monomorphémiques composés d'une pseudo-racine ne différant pas de la condition non reliée (cf. fig. 4).

Les deux résultats que nous avons obtenus semblent en revanche conformes aux prédictions que l'on peut dégager d'un modèle supralexical de représentation de la morphologie dans lequel les unités morphémiques sont situées à l'interface des niveaux lexical et sémantique de traitement. En effet, l'accès aux représentations lexicales n'étant pas médiatisé par l'activation d'unités morphémiques, ce modèle ne prédit, d'une part, 
aucune différence entre l'effet d'amorçage morphologique induit par une racine et celui induit par un mot morphologiquement complexe et, d'autre part, étant données les conditions d'amorçage masqué, aucune influence de la part des amorces contenant une pseudo-racine sur le traitement de cibles morphologiquement complexes.

EFFETS DE FRÉQUENCE DE SURFACE ET DE FREQUENCE CUMULÉE DANS LE PARADIGME D'AMORÇage MASQUÉ

Les mots morphologiquement complexes peuvent être décrits selon deux types de fréquence : leur fréquence de surface qui correspond à leur fréquence d'occurrence dans la langue (e.g., laitier) et leur fréquence cumulée qui est calculée en faisant la somme des fréquences de surface de tous les mots dérivés de la même racine (e.g., laitier + laitage + laiterie $+\ldots+$ lait). Parce que les deux modèles de représentation de la morphologie situent les effets d'amorçage morphologiques au niveau des unités morphémiques de traitement, différentes prédictions peuvent être formulées quant à la façon dont l'effet d'amorçage morphologique devrait interférer avec les effets des fréquences décrivant les mots morphologiquement complexes.

Dans le modèle supralexical, les unités morphémiques sont situées à l'interface des niveaux lexical et sémantique, l'accès aux représentations lexicales se fait donc immédiatement après la présentation d'un mot stimulus à l'entrée du système. Une des conséquences directes de ce mécanisme est que l'amplitude des effets d'amorçage morphologique devrait fortement dépendre de la fréquence de surface des mots amorces. En effet, dans ce type d'architecture, l'activation d'une unité morphémique dépend de l'activation de l'ensemble des représentations de mots qui contiennent cette unité. Ainsi, dans des conditions d'amorçage bref et masqué', on peut émettre l'hypothèse selon laquelle la représentation de mot qui va être la plus rapidement activée va correspondre au mot amorce dont le seuil d'activation est le plus bas (McClelland et Rumelhart, 1981). Ainsi, dans de telles conditions, le seuil d'activation des unités morphémiques ne devrait donc dépendre que de la fréquence de surface de l'amorce et non de sa fréquence cumulée.

En revanche, dans le cadre du modèle sublexical, les effets d'amorçage morphologique résultent principalement de l'emprunt répété de l'unité morphémique d'accès partagée par le mot amorce et le mot cible relié. Étant donné que dans le modèle sublexical, les unités morphémiques

1. Pour lesquelles une durée très brève du traitement de l'amorce est allouée au système. Nous ferions des prédictions différentes s'il s'agissait de conditions d'amorçage où l'amorce est présentée de façon non masquée et suffisamment longtemps pour que le sujet puisse la percevoir consciemment. 
d'accès médiatisent l'accès aux représentations des mots complexes, leur vitesse d'activation devrait uniquement dépendre de leur fréquence cumulée.

Une seconde série d'expérimentations utilisant le paradigme d'amorçage masqué a testé les effets respectifs des fréquences de surface et cumulée des mots amorces suffixés sur le traitement de mots cibles racines (Giraudo et Grainger, 2000). Une première expérience a montré que les mots amorces suffixés possédant une haute fréquence de surface (e.g., amitié-ami) produisaient des temps de décision lexicale significativement plus courts par rapport à ceux induits par des amorces orthographiques (e.g., amiral-ami) traduisant ainsi un effet d'amorçage morphologique différant d'un chevauchement formel entre l'amorce et la cible. En revanche, lorsque les mots suffixés présentés en amorce étaient de basse fréquence de surface, aucun effet d'amorçage n'a pu être observé, ni par rapport à la condition orthographique, ni par rapport à la condition non reliée (cf. fig. 5). Par ailleurs, la manipulation de la fréquence de surface d'amorces orthographiques n'a révélé aucun effet d'amorçage (cf. fig. 5) suggérant ainsi que l'effet d'amorçage observé dans la première expérience résulte de l'interaction entre la fréquence de surface de l'amorce morphologique et de la nature de la relation morphologique manipulée entre le mot amorce et le mot cible. Enfin, une troisième expérience manipulant la fréquence cumulée de mots amorces suffixés a mis en évidence que, si des effets d'amorçage morphologiques positifs étaient observés, l'amplitude de ces effets ne variait pas selon la fréquence cumulée du mot amorce (cf. fig. 6).

Ces résultats sont contraires aux données antérieures obtenues en Français par Grainger et ses collègues (1991). En effet, ces auteurs ont mis en évidence que la fréquence de surface n'interagissait pas avec les effets d'amorçage morphologique. Toutefois, dans ces expériences, la fréquence de surface des cibles covariait avec la fréquence de surface des amorces. En effet, dans ces expériences, les cibles de fréquence de surface moyenne étaient amorcées par des mots ayant une fréquence de surface basse tandis que les cibles de basse fréquence de surface étaient amorcées par des amorces ayant une fréquence de surface moyenne. Dans nos expériences, toutes les cibles correspondaient à des racines libres ayant une haute fréquence de surface. Or, la plus haute fréquence des cibles racines par rapport à celle des mots amorces morphologiquement reliés constitue une condition d'amorçage pour laquelle l'identification subséquente des mots cibles est la moins contrainte par les effets d'inhibition latérale opérés au niveau orthographique/phonologique (Segui et Grainger, 1990), permettant ainsi l'obtention d'effets de fréquence de surface provenant des mots amorces manipulés. Les résultats de Giraudo et Grainger (2000) précisent en outre que ces effets d'amorçage vont dans le sens d'un effet d'amorçage plus important dans le cas de mots amorces ayant une haute fréquence de surface que dans le cas de mots amorces possédant une basse fréquence de surface. 

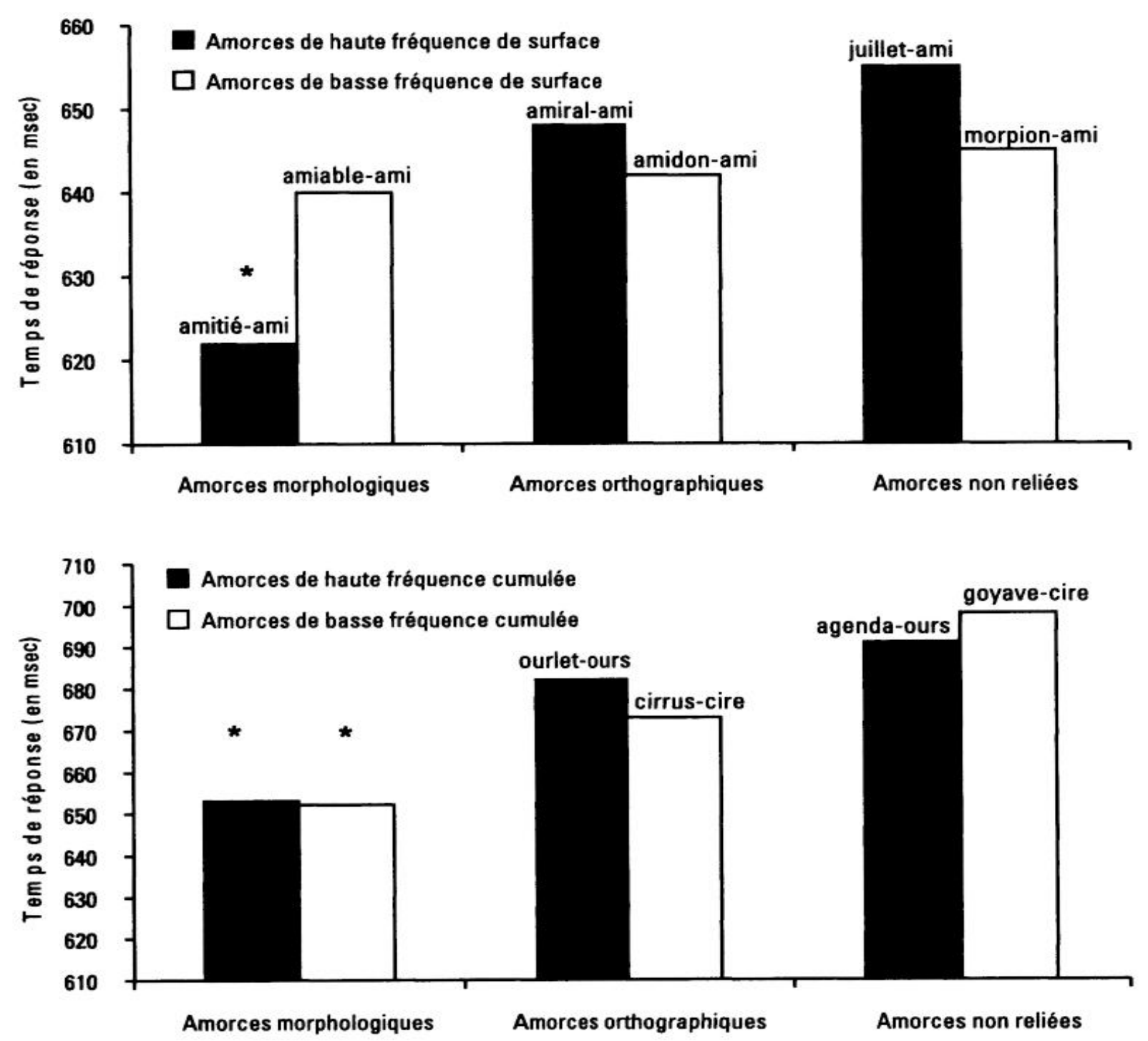

Fig. 5-6. - Principaux résultats obtenus par Giraudo et Grainger (2000). Latences de décision lexicale sur des mots cibles dérivés testés dans le paradigme d'amorçage masqué utilisant un temps d'exposition de l'amorce de $57 \mathrm{msec}$

* indique un effet d'amorçage significatif par rapport à la condition non reliée ( $p<.05$ par sujet et par item)

Main results obtained by Giraudo et Grainger (2000). Lexical decision times for derived target words tested in masked priming paradigm using 57 msec primes

* indicates a significant priming effect compared to the un-related condition ( $p<.05$ by subject and by item)

Par ailleurs, nos résultats ont montré qu'aucun effet de fréquence cumulée n'était observé sur les temps d'identification de racines libres. Ces résultats sont contraires aux effets de fréquence cumulée qui ont systématiquement été observés dans la littérature (e.g., Burani et Caramazza, 1987 ; Colé et al., 1997 ; Meunier et Segui, 1999). Néanmoins, à la différence des expériences présentées ici, ces études ont manipulé des cibles 
ayant des fréquences de surface relativement basse par rapport à celle de nos expériences et l'asymétrie de nos résultats par rapport aux précédents pourrait donc s'expliquer en termes de fréquence de surface des cibles utilisées.

Dans leur ensemble, ces résultats semblent contraires aux prédictions d'un modèle qui suppose que les unités morphémiques sont contactées avant les unités mots. Les données observées semblent plutôt en accord avec les prédictions d'un modèle dans lequel les unités morphémiques sont situées à l'interface des niveaux de représentation lexical et sémantique. En effet, le modèle supralexical prédit, dans le cadre du paradigme d'amorçage bref et masqué, des effets de fréquence de surface associés à une absence d'effet de fréquence cumulée.

\section{RÔLE ET REPRÉSENTATION DES AFFIXES EN MEMOIRE}

La linguistique française décrit les affixes dérivationnels comme correspondant à des morphèmes non autonomes (e.g., [-ier]) qui ont pour propriété de se combiner avec des morphèmes lexicaux (i.e., les racines telles que [lait-]) pour en indiquer la fonction syntaxique ou pour en changer la catégorie. Selon la place qu'ils occupent par rapport à la racine, on distingue les préfixes, placés avant la racine (e. g., prénom), des suffixes placés après la racine (e.g., laitier). Or, si de nombreuses études expérimentales se sont intéressées à la représentation de l'information morphologique en mémoire, la plupart de ces travaux s'est principalement focalisée sur la représentation des morphèmes racines. En effet, la littérature traitant des effets morphologiques ne comporte que très peu de données relatives au rôle et à la représentation des affixes dérivationnels (voir néanmoins Marslen-Wilson et al., 1994, et Marslen-Wilson et Zhou, 1999, pour une réflexion sur la problématique du statut des affixes en tant qu'entités indépendantes). En outre, si l'étude comparative du traitement des mots morphologiquement complexes par rapport à celui des mots contenant des pseudomorphèmes a occupé une large partie des études psycholinguistiques, l'objectif de ces travaux consistait principalement à éprouver la sensibilité du lecteur à la structure morphologique des mots et à mettre ou non en évidence l'emploi d'une procédure de décomposition morphologique prélexicale. Globalement, les travaux qui ont comparé les latences de traitement des mots complexes par rapport à celles des mots pseudo-complexes ont montré que la présence d'un pseudo-morphème dans une séquence de lettres, que celle-ci constitue un mot ou un pseudo-mot, occasionnait un coût supplémentaire de traitement par rapport à une condition neutre (Caramazza et al., 1988 ; Henderson, Wallis et Knight, 1984 ; Laine, 1995 ; Laudanna, Cermele et Caramazza, 1997 ; Pillon, 1998 ; Rubin, Becker et Freeman, 1979 ; Taft, 1981 ; Taft et Forster, 1975, 1976). Ce résultat fut interprété comme la preuve d'une analyse morphologique systématique 
prélexicale de toute séquence de lettres comportant un morphème potentiel, ceci favorisant l'hypothèse selon laquelle les mots plurimorphémiques sont accédés via l'activation de leurs morphèmes constituants.

Par ailleurs, trois études se sont intéressées au traitement et à la représentation des préfixes en prenant en compte les statistiques lexicales décrivant l'occurrence des mots préfixés et pseudo-préfixés dans la langue (rapport entre le nombre de mots préfixés comportant un préfixe donné et le nombre de mots pseudo-préfixés comportant cette même séquence de lettres comme pseudo-préfixe). La première concernant la productivité des mots préfixés et des mots pseudo-préfixés en Français (Pavard, 1983) souligne ainsi qu'il existe dans cette langue une forte proportion de mots pseudo-préfixés par rapport aux mots préfixés. En italien, l'étude de Laudanna, Burani et Cermele (1994) a mis en évidence que les non-mots contenant un préfixe ayant une forte probabilité de correspondre à un morphème prenaient plus de temps à être rejetés que les nonmots constitués d'un préfixe ayant une faible probabilité morphémique. Enfin, l'examen de l'Anglais et du Hollandais réalisé par Schreuder et Baayen (1994) a montré que la forte proportion des mots pseudo-préfixés par rapport à celle des mots préfixés constatée en général dans ces langues conférait à tout modèle de décomposition morphologique prélexical un caractère peu écologique étant donné le grand nombre d'impasses procédurales engendrées par ce type de mots. Dans leur ensemble, ces études statistiques ont suggéré, d'une part, que s'il existe une procédure de décomposition prélexicale, celle-ci doit tenir compte du caractère morphologique probable de toute séquence de lettres identique à un morphème et, d'autre part, qu'une procédure d'analyse morphologique systématique et obligatoire a une faible valeur heuristique dans les langues comportant un grand nombre de mots pseudo-complexes.

Une série d'expérimentations utilisant les paradigmes d'amorçage masqué et non masqué a tenté de déterminer si les affixes dérivationnels au même titre que les racines pouvaient être représentés explicitement en mémoire sous forme d'unités morphémiques indépendantes de traitement (Giraudo et Grainger, 2003). L'amorçage bref et masqué de mots préfixés et suffixés dérivés a été réalisé en manipulant trois types d'amorce : 1 / morphologique, où l'amorce correspondait à un mot affixé partageant le même affixe que la cible (e.g., surjet-survie; jupon-raton), 2 / orthographique, où l'amorce correspondait à un mot pseudo-affixé constitué d'une séquence de lettres identique à l'affixe contenu dans la cible (e.g., sureau-survie; melonraton) et 3) non reliée utilisant un mot ne partageant aucun trait commun avec la cible (e.g., goyave-survie; lilas-raton).

Les résultats ont mis en évidence un effet facilitateur des amorces préfixées sur les temps de décision lexicale des cibles préfixée par rapport à la condition pseudo-préfixée et la condition non reliée. Aucune différence n'a été observée entre la condition pseudo-préfixée et la condition non reliée nous assurant ainsi que l'effet positif d'amorçage observé entre deux mots 
préfixés relevait bien d'un effet de facilitation morphologique et non pas d'un simple partage formel entre l'amorce et la cible. En revanche, aucun effet d'amorçage n'a été observé pour les cibles suffixées quelle que soit la condition d'amorçage (cf. fig. 7 ).

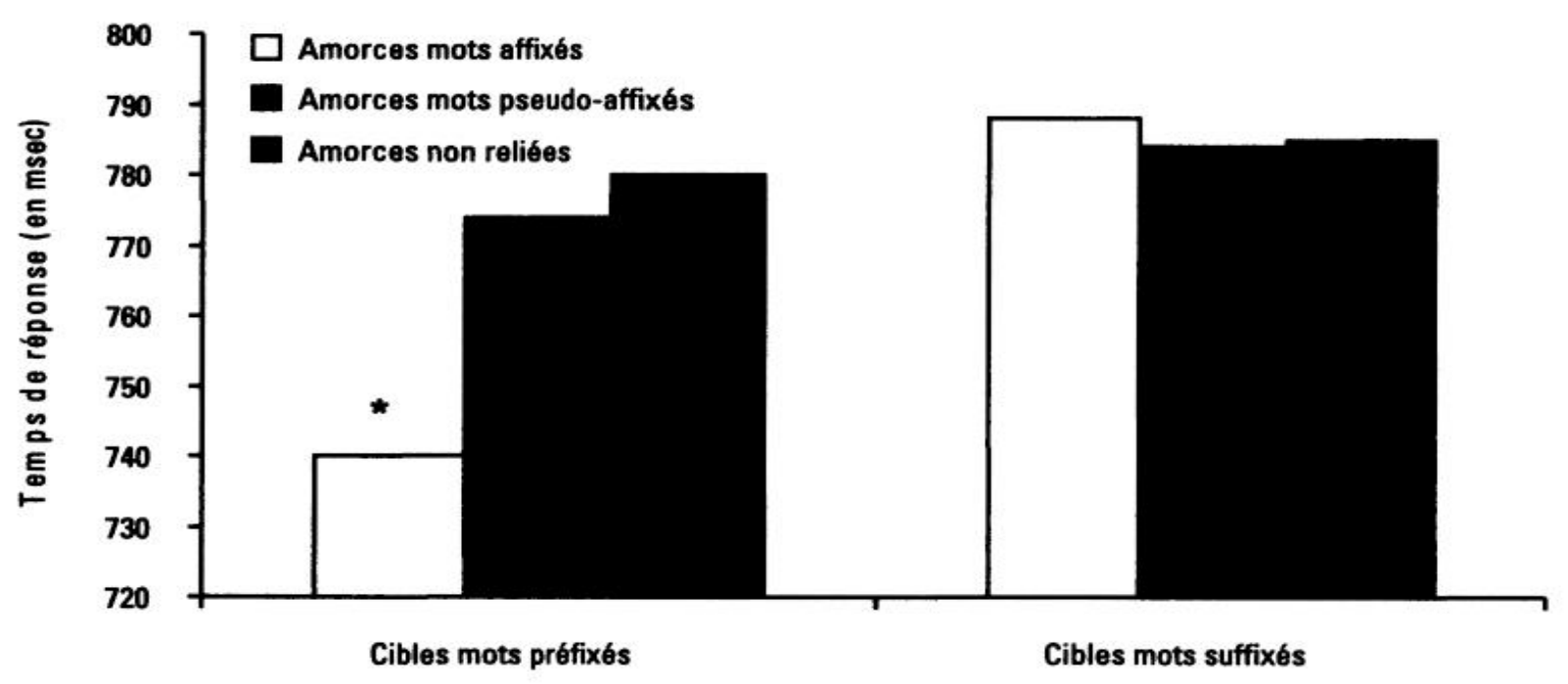

Fig. 7. - Principaux résultats obtenus par Giraudo et Grainger (2003). Latences de décision lexicale sur des mots cibles dérivés testés dans le paradigme d'amorçage masqué utilisant un temps d'exposition de l'amorce de $57 \mathrm{msec}$

* indique un effet d'amorçage significatif par rapport à la condition contrôle - orthographique ou non reliée - ( $p<.05$ par sujet et par item)

Main results obtained by Giraudo and Grainger (2003). Lexical decision times for derived target words tested in masked priming paradigm using 57 msec primes

*indicates a significant priming effect compared to the spelling or un-related control condition ( $p<.05$ by subject and by item)

Si l'on part du postulat que les effets d'amorçage correspondent à un effet de transfert d'activation entre la représentation du mot amorce et celle du mot cible, ce transfert s'effectuant via une représentation partagée par les représentations lexicales respectives de l'amorce et de la cible, ces résultats suggèrent que les morphèmes préfixes sont représentés dans le lexique sous la forme de représentations morphologiques explicites et indépendantes qui sont connectées par des liens excitateurs aux représentations lexicales constituées de ces morphèmes.

Par ailleurs, l'utilisation d'une condition contrôle orthographique nous a aussi permis de tester les prédictions respectives des deux architectures de représentation de l'information morphologique en mémoire. En effet, pour les mêmes raisons que celles invoquées dans la partie concernant les effets d'amorçage issus de la présentation d'un mot contenant une pseudo-racine, un modèle supralexical ne prédit pas d'effet d'amorçage entre un mot comportant un pseudo-affixe et un mot affixé alors que le modèle sublexical prédit au contraire que, dans des conditions d'amorçage bref et masqué, la 
présence d'une séquence de lettres pouvant potentiellement correspondre à un affixe devrait permettre l'activation d'une unité affixe qui à son tour ira activer les représentations lexicales de mots complexes constitués de cet affixe. Cela permettant, par conséquent, l'observation d'un effet d'amorçage entre un mot contenant un pseudo-affixe et un mot affixé, même s'il ne s'agit là que d'une pure relation formelle. Or les résultats expérimentaux ont révélé que seule la condition morphologique, c'est-à-dire réellement préfixée, produisait un effet d'amorçage significatif sur les temps de traitement des mots cibles préfixés.

Ce dernier résultat associé aux conclusions établies par Laudanna et ses collaborateurs (1994) et par Schreuder et Baayen (1994) selon lesquelles une décomposition morphologique prélexicale ne peut avoir de réalité psychologique étant donné la distribution des mots affixés et des mots pseudoaffixés dans la langue suggère une fois de plus que le modèle supralexical permet le mieux de rendre compte des effets d'amorçage morphologique. En effet, de par le locus supralexical des unités morphémiques, dans des conditions d'amorçage bref et masqué, le modèle que nous proposons ne fait aucunement l'hypothèse d'un traitement automatique et irrépressible de toute séquence de lettres pouvant correspondre à un morphème.

Les résultats que nous avons obtenus n'ont cependant pu mettre en évidence d'effet d'amorçage par le suffixe. Et une telle asymétrie entre le traitement des mots préfixés et celui des mots suffixés a déjà été observée dans plusieurs études en Français. Par exemple, Colé, Beauvillain et Segui (1989) ont montré que les latences de décision lexicale des mots préfixés et suffixés étaient affectées par la manipulation de la fréquence de surface ${ }^{1}$ de ces mots, alors que seuls les mots suffixés étaient sensibles à la manipulation de leur fréquence cumulée (voir aussi Meunier et Segui, 1999, pour le même constat en modalité auditive). Par ailleurs, les expériences de Beauvillain (1996) utilisant la technique des mouvements oculaires durant la lecture de mots préfixés et suffixés ont mis en évidence que, si la fréquence de surface des mots affectait la durée de deuxième fixation des mots préfixés et suffixés, l'effet de la fréquence cumulée se manifestait sur la durée de la deuxième fixation d'un mot préfixé et sur celle de première fixation d'un mot suffixé. Ces résultats suggèrent selon l'auteur que l'information morphologique est intégrée selon différents moments durant le processus de lecture, ce en fonction de la position de la racine dans le mot (Beauvillain, 1996). Colé et ses collaborateurs (1989) et Beauvillain (1996) ont proposé que l'asymétrie constatée entre le traitement des mots préfixés et suffixés relevait directement de la différence d'organisation morphologique de ces

1. La fréquence de surface d'un mot correspond au nombre d'occurrence de ce mot dans la langue.

2. La fréquence cumulée renvoie à la fréquence cumulée de la racine et de tous les mots formés à partir de cette dernière (i.e., lait + laitage + laitier + laiterie + ...). 
deux types de mots (affixe + racine $v$ sacine + affixe), cela impliquant que les mots suffixés et non pas les mots préfixés sont accédés via la représentation de leur racine. Au regard de cet ensemble de résultats, on peut supposer que le traitement des mots suffixés serait plutôt guidé par la représentation de leur racine (située en début de mot, la racine est encore plus prédominante en modalité auditive) alors que celui des mots préfixés pourrait être plutôt guidé par la représentation de leur préfixe.

Une autre interprétation de cette asymétrie de traitement des mots préfixés et des mots suffixés français peut aussi se faire d'un point de vue linguistique. Cette explication est directement issue de la description fonctionnelle que les linguistes font des préfixes par rapport aux suffixes (e.g., Gardes-Tamines, 1988). En effet, les descriptions de la linguistique différencient les phénomènes de préfixation et de suffixation du point de vue des changements sémantiques et morpho-syntaxiques que l'adjonction de ces deux types d'affixes produit sur la racine. L'ajout d'un préfixe à une racine n'entraîne jamais la création d'un nouveau mot dont la classe morpho-syntaxique differe de celle de la racine. De plus, les préfixes n'ont pas de fonction grammaticale, ils introduisent uniquement un changement de sens. Leur fonction est donc exclusivement sémantique. À l'inverse, les suffixes ont des fonctions linguistiques beaucoup plus diversifiées', ils ont des valeurs grammaticales et contrairement aux préfixes, l'ajout d'un suffixe peut entraîner des allomorphes de la racine (tels que plomb dans plombier). Ces différences fonctionnelles pourraient avoir des conséquences directes sur la façon dont ces informations sont représentées en mémoire.

\section{DISCUSSION GÉNÉRALE}

Deux modèles (supralexical et sublexical) incorporant un niveau de représentation explicite de la morphologie en mémoire ont été proposés. Ils different du point de vue du locus des unités morphémiques par rapport aux unités lexicales et aux unités sémantiques de traitement. Cette différence d'organisation de niveaux nous a permis de générer un ensemble de prédictions relatives aux effets $d$ 'amorçage morphologique, que nous avons testées au travers d'une série d'expérimentations utilisant le paradigme d'amorçage masqué associé à la tâche de décision lexicale (Giraudo et Grainger, $2000 ; 2001 ; 2003)$. Les résultats de ces expériences ont permis de

1. Le suffixe peut modifier la valeur de l'emploi d'une base sans changer totalement son sens (cas des diminutifs tels que -ette dans maisonnette). Le suffixe peut aussi restreindre l'aire de l'emploi de la base à laquelle il s'ajoute. Le produit de la combinaison va se restreindre à un champ particulier de la langue alors que l'emploi de la base seule est très varié (cas des indicateurs lexicaux tels que -ite dans appendicite qui appartient au vocabulaire médical). 
mettre en évidence : 1 / que seuls les mots amorces réellement morphologiquement complexes facilitaient le traitement de mots cibles par rapport à une condition non reliée et que l'amplitude de l'effet de facilitation morphologique était indépendante du type morphologique de l'amorce (i.e., racine libre ou mot morphologiquement complexe), 2 / que des effets d'amorçage morphologique facilitateurs étaient observés entre deux mots partageant le même préfixe, à la fois par rapport à une condition d'amorçage pseudo-préfixée et une condition d'amorçage non reliée et enfin 3 / que les effets d'amorçage morphologique sur le traitement de cibles racines libres de haute fréquence étaient modulés par la fréquence de surface des mots amorces mais pas par la fréquence cumulée les décrivant.

Ces données peuvent néanmoins aussi être interprétées dans des modèles qui supposent que les représentations lexicales mots morphologiquement complexes peuvent être contactées selon deux voies d'accès, une voie d'accès globale et une voie d'accès de décomposition morphologique. Les deux voies d'accès sont activées en parallèle et sont en compétition pour gagner le niveau lexical. Il est supposé en outre que la voie globale est généralement la plus rapide car elle ne nécessite pas procédure de décomposition (Caramazza et al., 1988 ; Frauenfelder et Schreuder, 1992 ; Schreuder et Baayen, 1995).

Tout comme le modèle supralexical, ce type de modèle prédit, dans le cadre des expériences ayant manipulé des mots amorces suffixés et des mots amorces racines, que ces deux types de mots ont une influence équivalente sur le traitement des mots cibles. Dans une architecture double voie, les deux types de mots amorces activent la voie d'accès globale tandis que seuls les amorces mots complexes activent en plus la voie de décomposition morphémique. Néanmoins, comme il est supposé dans ce type de modèle que la voie d'accès globale est toujours plus rapide que la voie d'accès morphémique, aucune différence n'est attendue entre une amorçage réalisé à l'aide d'un mot simple et celui réalisé avec un mot complexe, dans la mesure où les deux types d'amorces sont appariés en fréquence de surface (ce qui était le cas dans les expériences présentées). Par ailleurs, un modèle double voie ne prédit pas non plus d'effet d'amorçage lorsque l'amorce correspond à un mot constitué d'une pseudo-racine car, en effet, ce type de modèle suppose que seuls les mots réellement complexes activent, en plus de la voie globale, une voie d'accès de décomposition morphologique. Par conséquent la présentation subséquente d'un mot cible constitué d'une racine identique (e.g., lait) à la pseudo-racine de l'amorce (e.g., laitue) ne devrait pas être facilitée car la voie d'accès morphémique n'aura pu être préalablement activée. Concernant ce dernier point, on peut néanmoins s'interroger sur les raisons pour lesquelles dans ces conditions d'amorçage brèves et masquées le système ayant détecté une séquence de lettres pouvant correspondre à un morphème n'entamerait pas une procédure de décomposition morphologique. Néanmoins comme récemment souligné par Longtin, Segui et Hallé (2003), il semblerait que la présence de deux pseu- 
do-morphèmes soit nécessaire à la mise en place d'une telle procédure. En effet, ces auteurs ont mis en évidence à l'aide du paradigme d'amorçage masqué que des effets d'amorçage pouvaient être observés entre un mot amorce pseudo-dérivé et un mot cible racine libre (tel que baguette est constitué de la pseudo-racine bagu- et du pseudo-suffixe -ette mais ne constitue en aucun cas un mot morphologiquement complexe pouvant être relié au mot bague). Des résultats identiques ont été mis en évidence en anglais (Rastle et Davis, 2003 ; Rastle, Davis et $\mathrm{New}$, sous presse). Ces résultats ont été interprétés par les auteurs à la fois dans un modèle double voie et dans un modèle sublexical. Toutefois, un modèle supralexical peut tout aussi bien intégrer ce type d'effet d'amorçage en supposant qu'une amorce pseudo-dérivée telle que baguette va activer un ensemble de mots globaux au niveau lexical dont des mots complexes tels que bague, baguier, bagueur... Ces mots complexes étant reliés à des morphèmes situés au niveau supérieur vont alors activer ces derniers et la reconnaissance d'un mot cible tel que bague en sera subséquemment facilitée (voir Giraudo et Grainger, 2003, pour une discussion plus approfondie).

Concernant les effets d'amorçage que nous avons obtenus lorsque des morphèmes affixes étaient manipulés, il semble qu'un modèle double voie qui présuppose l'activation d'une voie de décomposition morphologique pré-lexicale ne puisse, tout comme un modèle sublexical, expliquer l'absence d'effet d'amorçage entre deux mots suffixés alors que des effets positifs d'amorçage ont pu être observés entre deux mots partageant le même préfixe. La reconnaissance d'un mot cible suffixé devrait être facilitée par la présentation préalable d'un mot suffixé partageant le même suffixe car ce dernier aura permis l'activation de la voie d'accès de décomposition morphémique. Par conséquent, la reconnaissance du mot cible suffixé devrait bénéficier de la préactivation de son suffixe. En revanche, on peut tout à fait supposer dans un modèle supralexical, qui inclut un niveau de représentation morphologique situé à l'interface des niveaux lexical et sémantique, que les unités morphémiques vont émerger en mémoire suite aux multiples interactions entre les niveaux lexical et sémantique que génèrent les mots morphologiquement complexes. Cela suppose donc que, lors de l'acquisition du langage, les unités morphémiques sont dans un premier temps des unités émergentes (de la même manière que cela est supposé dans les modèles PDP de la reconnaissance de mots, voir Plaut et Gonnerman, 2000 ; Seidenberg et Gonnerman, 2000) qui vont a vec l'expérience de la langue se fixer sous une forme explicite et indépendante en mémoire à long terme. Par conséquent, on peut faire l'hypothèse que seules les unités transparentes d'un point de vue formel et sémantique seront représentées en mémoire. En français, les préfixes étant beaucoup plus transparents sémantiquement que les suffixes, on peut supposer que seuls ces derniers posséderont une représentation au niveau morphologique. Mots préfixés et mots suffixés pourraient différer ainsi du point de vue de leur représentation morphologique en mémoire, les uns étant représentés à la fois du point 
de vue de leur morphème de base et de leur préfixe, les autres ne pouvant activer que leur base au niveau de représentation morphologique. Des recherches supplémentaires orientées dans cette direction semblent donc nécessaires afin d'expliquer les différences de traitement entre ces deux catégories de mots complexes.

Enfin, la troisième partie des recherches présentées concernait l'influence des fréquences de surface et cumulée des mots amorces reliés morphologiquement sur le traitement de mots cibles. Les résultats ont mis en évidence une influence de la fréquence de surface associée à une absence d'effet de la fréquence cumulée des mots amorces sur le traitement visuel des cibles. Dans ce cadre, un modèle double voie fait les mêmes prédictions qu'un modèle supralexical : la fréquence de surface est déterminante dans la vitesse d'accès au lexique car dans ce type de modèle la voie d'accès globale, qui est sensible à la fréquence de surface des mots, est toujours plus rapide que la voie d'accès morphémique. D'autre part, à fréquence de surface équivalente aucune différence d'effet $n$ 'est attendue entre une amorce de haute fréquence cumulée et une amorce de basse fréquence cumulée car la voie d'accès globale est toujours efficace.

En conclusion, bien qu'un modèle sublexical ou un modèle voie puisse expliquer une grande partie des données présentées dans nos expériences et au travers de la littérature, seule une architecture supralexicale, dans laquelle les unités morphémiques sont situées à l'interface des niveaux de représentation des unités lexicales et sémantiques, et fonctionnant comme unités d'organisation du lexique mental en termes de familles morphologiques, semble offrir une flexibilité suffisante pour permettre de représenter au niveau de représentation morphologique certains morphèmes et pas d'autres sur la base de leurs régularités formelles et sémantiques dans la langue (comme par exemple des préfixes et pas des suffixes). Les recherches que nous menons actuellement s'inscrivent dans la cadre de l'élaboration de ce type de modèle en s'attachant à préciser quels sont les autres facteurs qui sous-tendent le traitement morphologique.

\section{RESUME}

La plupart des travaux actuels tendent à donner aux informations morphologiques un rôle essentiel du point de vue de l'accès lexical, néanmoins de nombreuses controverses subsistent concernant la façon où ces informations sont représentées en mémoire à long terme. Si l'on se place dans le cadre de modèles hiérarchiques de la reconnaissance de mots, dans lesquels les codes lexicaux sont traduits en codes orthographiques et ou phonologiques décrivant les mots entiers, puis en codes sémantiques, deux localisations peuvent être proposées pour des représentations morphologiques : avant les représentations de mots (hypothèse sublexicale) ou après ces représentations (hypothèse supralexicale). Selon l'hypothèse sublexicale, la reconnaissance d'un mot engendre systématiquement une procédure d'analyse de ses constituants morphémiques avant 
que celui-ci ne puisse être identifíé selon sa forme globale. En revanche, selon l'hypothèse supralexicale, ce sont les représentations globales des mots qui, une fois activées, permettent l'activation des représentations morphémiques situées d̀ un niveau supérieur, ces dernières imposant ainsi aux représentations lexicales une organisation en termes de familles morphologiques. Cet article présente un ensemble de données récentes, obtenues en français et à l'aide du paradigme d'amorçage masqué associé à la tâche de décision lexicale, qui suggèrent que les informations morphologiques dérivationnelles sont représentées à un niveau supralexical de traitement.

Mots clés : reconnaissance de mots, information morphologique, modèle supralexical

\section{REFERENCES}

Beauvillain C. - (1996) The integration of morphological information and whole-word form information during eye fixations on prefixed and suffixed words, Journal of Memory and Language, 35, 801-820.

Bentin S., Feldman L. B. - (1990) The contribution of morphological and semantic relatedness to repetition priming at short and long lags : Evidence from Hebrew, The Quarterly Journal of Experimental Psychology, 42A, 693711.

Boudelaa S., Marslen-Wilson W. D. - (2000) Morphological units in Arabic mental lexicon, Cognition, 81 (1), 65-92.

Butterworth B. - (1983) Lexical Representation, Language Production, 2, 257 295.

Caramazza A., Laudanna A., Romani C. - (1988) Lexical access and inflectional morphology, Cognition, 28, 297-332.

Colé P., Beauvillain C., Segui, J. - (1989) On the representation and processing of prefixed and suffixed words : A differential frequency effect, Journal of Memory and Language, 28, 1-13.

Colé P., Segui J., Taft M. - (1997) Words and morphemes as units for lexical access, Journal of Memory and Language, 37, 312-330.

Deutsch A., Frost R., Forster K. I. - (1998) Verbs and nouns are organized and accessed differently in the mental lexicon : Evidence from Hebrew, Journal of Experimental Psychology : Learning, Memory, and Cognition, 24, 1238-1255.

Deutsch A., Frost R., Pollatsek A., Rayner,K. - (2000) Early morphological effects in word recognition in Hebrew : Evidence from parafoveal preview benefit, Language and Cognitive Processes, 15 (4/5), 487-506.

Drews E., Zwitserlood P. - (1995) Morphological and orthographic similarity in visual word recognition, Journal of Experimental Psychology : Human Perception and Performance, 21, 1098-1116.

Feldman L. B. - (1991) The contribution of morphology to word recognition, Psychological Research, 53, 33-41.

Feldman L. B., Bentin S. - (1994) Morphological analysis of disrupted morphemes : Evidence from Hebrew, The Quarterly Journal of Experimental Psychology, 47A (2), 407-435.

Feldman L. B., Soltano E. G. - (1999) Morphological priming : The role of prime duration, semantic transparency, and affix position, Brain and Language, 68, 33-39. 
Forster K. I., Davis C. - (1984) Repetition priming and frequency attenuation in lexical access, Journal of Experimental Psychology : Learning, Memory, and Cognition, 10, 680-698.

Forster K. I., Davis C., Schoknecht C., Carter, R. - (1987) Masked priming with graphemically related forms : Repetition or partial activation ?, The Quarterly Journal of Experimental Psychology, 39A, 211-251.

Frauenfelder U., Schreuder R. - (1992) Constraining psycholinguistic models of morphological processing and representation : The role of productivity, in G. Booij et J. Van Marle (Edit.), Yearbook of morphology, Amsterdam, Kluwer.

Frost R., Deutsch A., Forster K. I. - (2000) Decomposing morphologically complex words in non linearmorphology, Journal of Experimental Psychology : Learning, Memory, and Cognition, 26, 751-765.

Frost R., Deutsch A., Gilboa O., Tannenbaum M., Marslen-Wilson W. D. (2000) Morphological priming : Dissociation of phonological, semantic, and morphological factors, Memory and Cognition, 28 (8), 1277-1288.

Frost R., Forster K. I., Deutsch A. - (1997) What can we learn from the morphology of Hebrew ? A masked priming investigation of morphological representation, Journal of Experimental Psychology : Learning, Memory, and Cognition, 23 (4), 1-28.

Gardes-Tamine J. - (1988) La grammaire : phonologie, morphologie, lexicologie, t. 1, Paris, Armand Colin.

Giraudo H., Grainger J. - (2000) Effects of prime word frequency and morphemic frequency in masked morphological priming, Language and Cognitive Processes, 15 (4/5), $421-444$.

Giraudo H., Grainger J. - (2001) Priming complex words : Evidence for supralexical representation of morphology, Psychonomic Bulletin \& Review, 8 (1), 127-131.

Giraudo H., Grainger J. - (2003) On the role of derivational affixes in recognizing complex words : Evidence from masked priming, in $R$. H. Baayen et R. Schreuder (Edit.), Morphological structure in language processing, Berlin, Mouton de Gruyter.

Grainger J., Colé P., Segui J. - (1991) Masked morphological priming in visual word recognition, Journal of Memory and Language, 30, 370-384.

Grainger J., Jacobs A., M. - (1998), Localist connectionist approaches to human cognition, Mahwah, NJ, Erlbaum.

Henderson L., Wallis J., Knight D. - (1984) Morphemic structure and lexical access, in H. Bouma et D. Bouwhuis (Edit.), Attention and Performance, $X$, p. 221-224, Hillsdale, NJ, Lawrence Erlbaum Associates Inc.

Holmes V. M., O'Regan J., K. - (1992) Reading derivationnally affixed French words, Language and Cognitive Processes, 7, 163-192.

Laine M. - (1995) Lexical status of inflectional and derivational suffixes : Evidence from Finnish, Scandinavian Journal of Psychology, 36.

Laudanna A., Burani C., Cermele, A. - (1994) Prefixes as processing units, Language and Cognitive Processes, 9, 295-316.

Laudanna A., Cermele A. Caramazza A. - (1997) Morpho-lexical representations in naming, Language and Cognitive Processes, 12 (1), 49-66.

Longtin C. M. Segui J.,, Hallé P. A. - (2003) Morphological priming without morphological relationships, Language and Cognitive Processes, 18, 313-334.

Manelis L., Tharp D. - (1977) The processing of affixed words Memory \& Cognition, 5, 690-695. 
Marslen-Wilson W. D., Komisarjevsky-Tyler L. K., Waksler R., Older L. (1994) Morphology and meaning in the English mental lexicon, Psychological Review, 101, 3-33.

McClelland J. L. Elman J. L. - (1986) The TRACE model of speech perception, Cognitive Psychology, 18, 1-86.

Meunier F., Segui J. - (1999) Frequency effects in auditory word recognition : The case of suffixed words, Journal of Memory and Language, 41, 327-344.

Napps S. E. - (1989) Morphemic relationships in the lexicon : Are they distinct from semantic and formal relationships?, Memory \& Cognition, 17, 729-739.

Napps S. E., Fowler C. A. - (1987) Formal relationships among words and the organization of the mental lexicon, Journal of Psycholinguistic Research, 16, 257-272.

Pastizzo M. J., Feldman L. B. - (2002) Discrepancies between orthographic and unrelated baselines in masked priming undermine a decompositional account of morphological facilitation, Journal of Experimental Psychology : Learning, Memory, and Cognition, 28 (1), 244-249.

Pavard B. - (1983) Traitement perceptif des mots affixés : mise en évidence d'un contrôle cognitif, L'Année psychologique, 83, 443-464.

Plaut D. C., Gonnerman L. G. - (2000) Are non-semantic effects incompatible with a distributed connectionisy approach to lexial processing ?, Language and Cognitive Processes, 15 (4/5), 507-537.

Pillon A. - (1998) The pseudoprefixation effect in visual word recognition : A true-neither strategic nor orthographic-morphemic effect, The Quarterly Journal of Experimental Psychology, 51, 85-120.

Rastle K., Davis M. H. - (2003) Structural segmentation in early visual word recognition. Communication présentée au Third international workshop on morphological processing, Aix-en-Provence, 11-14 june, France.

Rastle K., Davis M. H., New B. (sous presse) The broth in my brother's brothel : Morpho-orthographic segmentation in visual word recognition, Psychonomic Bulletin and Review.

Rastle K., Davis M. H., Marslen-Wilson W. D., Tyler, K. L. - (2000) Morphological and semantic effects in visual word recognition : A time-course study, Language and Cognitive Processes, 15 (4/5), 507-538.

Rubin G. S., Becker C. A., Freeman R. H. - (1979) Morphological structure and its effects on visual word recognition, Journal of Verbal Learning and Verbal Behaviour, 18, 757-767.

Schreuder R., Baayen R. H. - (1994) Prefix stripping re-revisited, Journal of Memory and Language, 33, 357-375.

Schreuder R., Baayen R. H. - (1995) Modelling morphological processing. In L. B. Feldman (Edit.), Morphological aspects of language processing, p. 131 154, Hillsdale, NJ, Lawrence Erlbaum Associates Inc.

Schriefers H., Zwitserlood P., Roelofs A. - (1991) The identification of morphologically complex spoken words : Continuous processing or decomposition?, Journal of Memory and Language, 30, 26-47.

Segui J., Zubizarreta M.-L. - (1985) Mental representation of morphologically complex words and lexical access, Linguistics, 23, 759-774.

Seidenberg M. S., Gonnerman L. M. - (2000) Explaining derivational morphology as a convergence of codes, Trends in Cognitive Sciences, 4 (9), 353-361.

Stolz J., A. Besner D. - (1998) Levels of representation in visual word recognition. A dissociation between morphological and semantic processing, Jour- 
nal of Experimental Psychology : Learning, Memory, and Cognition, 24, 1642-1655.

Taft M. - (1981) Prefix stripping revisited, Journal of Verbal Learning and Verbal Behaviour, 20, 289-297.

Taft M., Forster K. I. - (1975) Lexical storage and retrieval of prefixed words, Journal of Verbal Learning and Verbal Behaviour, 14, 638-647.

Taft M., Forster K. I. - (1976) Lexical storage and retrieval of polymorphemic and polysyllabic words, Journal of Verbal Learning and Verbal Behavior, 15, 607-620.

Taft M. - (1994) Interactive-activation as a framework for understanding morphological processing, Language and Cognitive Processes, 9, 271-294.

Zwitserlood P. - (1994) The role of semantic transparency in the processing and the representation of Dutch compounds, Language and Cognitive Processes, 9 (3), 341-368. 